Methods The "exposed" group consisted of patients with MTBI ( $n=689$ at initial assessment) and the "unexposed" were patients with non-head injuries $(n=1318)$. All were drawn from five emergency departments in Southern California. The loss occurred over a 3-month period, when a second telephone survey was administered.

Results While the overall loss-to-followup rate was $37.8 \%$, participant dropout occurred differentially (34.1\% among MTBI patients vs $39.7 \%$ among the non-head-injured). A logistic regression model found several associated factors: age, education, race/ethnicity, employment and mode of injury, with assault having the highest odds of loss-to-followup (OR 2.23, 95\% CI 1.46 to 3.39). Comparisons between unweighted versus attrition-weighted values resulted in minimal differences.

Conclusion Although it is preferable to minimize loss-tofollowup through retention strategies and incentives (perhaps with additional focus on those factors identified in this study), employing attrition-weights may provide useful information.

\title{
0960 LOSS-TO-FOLLOW UP FACTORS IN EMERGENCY DEPARTMENT-TREATED MILD TRAUMATIC BRAIN INJURIES
}

PHsu*, J Kraus, A A Afifi, FVaca, K Schaffer Correspondence: UCLA, 10960 Wilshire Blvd. \#1550, Los Angeles, CA 90024, USA

10.1136/ip.2010.029215.960

Introduction The number of subjects in a prospective cohort study often decreases over time (sometimes substantially). This "loss-to-followup" can lead to bias or distortion of results, and in studies of less serious conditions or injuries such as mild traumatic brain injuries (MTBI), may be especially problematic. This manuscript reports on the factors associated with the loss-to-followup that occurred in a cohort of emergency department-treated mild traumatic brain injuries, and on the use of attrition weights to adjust for the potential associated bias. 